\title{
Heat shock proteins as the efficient vehicle in cancer
}

\author{
Sahar Hosseinzadeh ${ }^{1,2}$, Amin Daemi ${ }^{1,3}$, Azam Bolhassani ${ }^{1}$ \\ 1. Molecular Immunology and Vaccine Research Lab, Pasteur Institute of Iran, Tehran, Iran 2. Islamic Azad University of \\ Pharmaceutical Science, Tehran, Iran 3. International Branch of Shahid Beheshti Medical University, Tehran, Iran \\ Correspondence: Azam Bolhassani. Address: Molecular Immunology and Vaccine Research Lab, Pasteur Institute of \\ Iran, Tehran, Iran. Telephone: 98-216-6496-560. Fax: 98-216-6465-132. \\ E-mail: azam_bolhassani@yahoo.com
}

Received: March 25, 2012

Accepted: May 6, 2012

Published: June 1, 2012

DOI : $10.5430 /$ jst.v2n3p47

URL: http://dx.doi.org/10.5430/jst.v2n3p47

\section{Abstract}

Successful vaccine development requires knowing which adjuvants to use and how to formulate adjuvants and antigens to achieve stable, safe and immunogenic vaccines. There is growing literature on immuno-regulatory and adjuvant functions of heat shock proteins (HSPs) in the development of preventive and therapeutic vaccines against cancer and infectious diseases. These extremely conserved molecules associate with antigenic peptides from tumor, virus and intracellular bacteria, present these loaded antigens to both MHC class I and class II molecules and activate specific T cells. This review aims to summarize the data on the HSP roles as the efficient tools in cancer. The ability of heat shock proteins to chaperone peptides (e.g., antigenic peptides); interact with antigen presenting cells (APCs) through a receptor; stimulate APCs to secrete inflammatory cytokines; and mediate maturation of dendritic cells, permit the utilization of these proteins to develop a new generation of prophylactic and therapeutic vaccines against cancers and infectious diseases. Furthermore, some cancers demonstrate elevated levels of HSPs and their expression has been associated with cell proliferation and disease prognosis. New adjuvant development is needed to identify novel combinations of adjuvants and formulations capable of inducing strong, long lasting humoral and cellular immune responses in humans. Numerous challenges remain related to adjuvant development. Among efficient adjuvants, it has been shown that heat shock proteins induce cross-presentation of antigens by dendritic cells (DC) as well as DC maturation. These properties make HSP-antigen complexes good candidates to prime CD8 $+\mathrm{T}$ cell responses against tumor-associated antigens. Moreover, these conserved proteins can be used as diagnostic biomarkers in various cancers.

\section{Key words}

Heat shock protein, Cancer, Preventive/therapeutic vaccine, Adjuvant, Biomarker

\section{Introduction}

Heat shock proteins (HSPs) are members of a number of families of stress-induced proteins, whose main intracellular functions are as molecular chaperones ${ }^{[1]}$. HSPs are classified into different families on the basis of their apparent molecular size, structure and function. Those families include HSP100 and higher molecular weight (MW), HSP90, HSP70, HSP60 (chaperonin) and small HSP ${ }^{[2]}$.

Heat shock proteins (HSPs) are potent inducers of an antigen-specific immunological response. HSPs are capable of promoting antigen presentation of chaperoned peptides through interaction with the receptors on APCs (i.e., antigen 
cross-presentation). In previous animal studies, immunization with HSP-peptide complexes purified from tumor cells provided protection against tumors from which the complexes were derived. In addition, the utilization of this vaccine to treat mice with established tumors could slow the rate of tumor growth1. In clinical trials, autologous tumor-derived Gp96-peptide complexes were used to treat a variety of malignancies, including melanoma, colorectal cancer, and renal cell carcinoma with immunological and clinical responses in a subset of patients. But, however, the clinical response was silent in the randomized phase III trial. These results suggested a need for enhancement in the potency of such vaccines ${ }^{[1]}$. Recently, it has been found out that immunization with antigens genetically fused to HSP70 elicits strong and long-lived humoral and cellular immune responses in the absence of adjuvant. In addition, HSP70 fusion proteins elicited antigen-specific CTL responses and protected mice from tumor challenge. Indeed, chemical conjugation or genetic fusion of antigens to mycobacterial HSP70 has created potent immunogens that can elicit MHC class I-restricted, CD8+ cytotoxic $\mathrm{T}$ cell responses sufficient to mediate rejection of tumors expressing the fusion partner ${ }^{[2]}$. Most of this work has been done in mice, however, HSP conjugate vaccines have elicited immune responses in monkeys and HSP fusion vaccines are being tested in humans. The objective of this review is to summarize the HSP functions in cancers.

\section{General biochemical aspects of HSPs in cancer}

Heat shock proteins are of potential interest to cancer researchers. Some researchers have focused on the use of heat shock proteins in the treatment of cancer. The researchers have shown that HSPs may be involved in binding protein fragments from dead malignant cells and presenting them to the immune system ${ }^{[2]}$. HSPs are highly abundant proteins in eukaryotic cells that are highly conserved during evolution and protect cells from damage through their function as molecular chaperones. Some cancers demonstrate elevated levels of HSPs (e.g., Hsp70), and their expression has been associated with cell proliferation, disease prognosis, and resistance to chemotherapy ${ }^{[3]}$. HSPs represent about $1 \%-2 \%$ of total proteins in un-stressed cells and increase to $4 \%-6 \%$ of cellular proteins under stress. Under stress conditions, HSPs are rapidly induced through transcription and translation mechanisms. The transcription of HSP genes is regulated by a family of heat shock transcription factors (HSFs) including HSF ${ }^{[4]}$. Recent reports demonstrated that HSF1 plays a key role in the development of tumors associated with activation of Ras or inactivation of p53 and HSF1 inactivation inhibits the progression of a wide spectrum of cancers ${ }^{[4]}$.

Among heat shock proteins, HSP27, HSP60, HSP70 and HSP90 have been investigated in clinical settings using samples of human cancers. During carcinogenesis, the expression levels of HSPs can either increase or decrease. For example, the expression of HSP27, HSP60, HSP70 and HSP90 was increased in human bladder cancers ${ }^{[5]}$. Also, HSP70 was over-expressed in malignant melanoma and in renal cell cancer ${ }^{[6]}$. In other studies, altered expression of calreticulin (CRT) has been detected in melanoma and in liver, bladder, prostate, lung, pancreatic and breast cancers ${ }^{[7]}$.

HSP70 and HSP90 were known to act as anti-apoptotic factors ${ }^{[6]}$. Hsp90 participates in many key processes in oncogenesis such as self-sufficiency in growth signals, stabilization of mutant proteins, angiogenesis and metastasis. HSP60 is also a mitochondrial protein involved in activation of apoptosis. Recently, the role of small heat shock proteins (e.g., HSP27, $\alpha$ A/B-crystallins) has been widely recognized in cancer research. Small HSPs are tumor protective molecules via various mechanisms such as: Oxidative stress, prevention of protein denaturation, anti-apoptotic activity and likely direct suppression of the immune system ${ }^{[6]}$. Briefly, the properties of important HSP families are individually described:

\section{HSP70}

Human cells include several HSP70 family members; the stress-inducible HSP70 (HSP72 or HSPA1), the constitutively expressed heat shock cognate 70 (HSC70, HSP73 or HSPA8) in the cytosol and nucleus, mitochondrial HSP70 (Grp75, Mortalin or HSPA9) and glucose regulated protein 78 (Grp78, HSPA5) in the endoplasmic reticulum ${ }^{[4]}$. Under normal conditions, HSP70 plays multiple roles such as the folding of newly synthesized proteins, the transport of proteins and vesicles, the assembly and dissociation of multi-protein complexes and the degradation of denature proteins. HSP70 is also 
powerful anti-apoptotic protein that acts at different key points, affecting both the extrinsic and intrinsic pathway of apoptosis. HSP70 regulates apoptosis by inhibiting stress-induced signals, by preventing mitochondrial membrane permeabilization and by suppressing caspase activation and DNA fragmentation ${ }^{[4]}$.

HSP70 is expressed at high levels in a wide spectrum of cancer cells and HSP70 expression has been routinely associated with poor prognosis. The exact role of the HSP70 in cancer remains to be elucidated. However, in cancer cell, HSP70 over-expression is thought to provide a survival advantage due to its ability to inhibit apoptosis and senescence. Many studies reported that HSP70 over-expression has also been correlated with therapeutic resistance due to the reduced activation of ERK, NF- $\mathrm{kB}$ and JNK pathways ${ }^{[4]}$.

\section{HSP90}

HSP90 is a highly abundant and evolutionarily conserved protein in the all eukaryotic cell. Five HSP90 isoforms have been identified to date such as the two major cytoplasmic isoforms, HPS90 $\alpha$ (HSPC1) and HPS90 $\beta$ (HSPC3), endoplasmic reticulum localized glucose regulated protein 94 (Grp94), mitochondrial tumor necrosis factor receptor-associated protein 1 (TRAP1) and membrane-associated HSP90N. Despite their different cellular localization, these isoforms have a similar structure and function as chaperone ${ }^{[4]}$.

HSP90 functions as a part of a multi-chaperone complex via association with a variety co-chaperons and client proteins that rely on the complex for maturation and stability. More than 200 client proteins have been identified so far. Its client proteins include BCL-ABL, SRC, HER2, EGFR, CRAF, BRAF, AKT, MET, VEGFR, FLT3, androgen and estrogen receptor, hypoxia-inducible factor (HIF)- $1 \alpha$, and telomerase that are directly involved in malignancy and mutated oncogenic proteins that are required for the transformed phenotype. These include important proteins in breast cancer progression such as HER2 and c-SRC. Indeed, HSP90 over-expression has been observed in a variety of human malignancies including breast cancer. In addition, HSP90 may inhibit apoptosis through numerous interactions ${ }^{[4]}$.

\section{HSP27}

HSP27 is a member of the small HSPs family that acts as an ATP-independent chaperone and mainly localized in the cytosol. They are potent mediator of protein folding and also involved in architecture of cytoskeleton, cell migration, cell growth/differentiation, and tumor progression. HSP27 also has anti-apoptotic property. High levels of HSP27 have been observed in many cancer cells including breast carcinoma as compared to normal cells ${ }^{[4]}$. Recently, several studies demonstrated that the over-expression of HSP27 seems to be correlated with increased resistance to chemotherapeutic drug-induced apoptosis in cancer cells ${ }^{[4]}$.

\section{Calreticulin}

Calreticulin (CRT) is a chaperone protein located in the lumen of the endoplasmic reticulum. The association of calreticulin with pathological conditions such as autoimmune disorders and certain types of cancer has been reported. The data suggest that calreticulin expression is associated with more advanced tumors and is a potential prognostic biomarker (e.g., breast cancer) ${ }^{[8]}$. Interestingly, lower expression of calreticulin was observed in human colonic cancer cell lines than in preparations of isolated human normal colonic epithelial cells. Some results also showed that calreticulin is likely to play a pivotal role in the differentiation of human colonic adenocarcinomas ${ }^{[9]}$. Furthermore, the studies have indicated that the recombinant calreticulin NH2 domain (Amino acids 1-180), but not calreticulin P domain (Amino acids 181-189), can inhibit endothelial cell proliferation in vitro and angiogenesis in vivo ${ }^{[10]}$.

Liu et al. showed that soluble calreticulin (sCRT) concentration in sera of lung cancer patients is higher than that in sera of healthy individuals, and CRT expression level on lung cancer cell membrane is associated with tumor pathological classification and grade. These findings suggest that CRT may be used as a biomarker in lung cancer prediction and diagnosis ${ }^{[11]}$. 


\section{I mmunological properties of HSPs in vaccine design}

Several studies have suggested that HSPs can interact with and inhibit both intrinsic and extrinsic pathways of apoptosis at multiple sites. The authors have shown that inhibition of HSPs induce apoptotic cell death in cancer cells suggesting that inhibition of HSPs has a potential to use as novel anti-cancer therapy, either as mono-therapy or in combination with other chemotherapeutic agents. Besides the anti-apoptotic role of HSPs, recent studies demonstrate that they play a role in the generation of anti-cancer immunity, suggesting the use of this property of HSPs in the design of anti-cancer vaccines ${ }^{[12]}$. There are various experiments in order to present immunological properties of HSPs and their use in vaccine development as follows.

In a study, the relative immunogenicities of gp96, hsp90, and hsp70 derived from the Meth A sarcoma have been compared. The proteins gp96 and hsp70 were observed to be highly and equally immunogenic, whereas the immunogenicity of hsp90 was approximately $10 \%$ of that of gp96 or hsp70. These data suggested that the poor immunogenicity of hsp90 results from its lack of a measurable ATPase activity, which has been implicated in the ability of HSPs to transfer peptide to acceptor molecules ${ }^{[13]}$.

Heat shock proteins including calreticulin (CRT), HSP70 and gp96, have been shown to act as potent immuno-adjuvant to enhance antigen specific tumor immunity (Figure 1). Several studies have shown that gp96 as an adjuvant induces antigen specific $\mathrm{T}$ cell responses against cancer and infectious diseases. Heat shock protein gp96 is a highly conserved and mono-morphic glycoprotein in the endoplasmic reticulum. It functions as molecular chaperone and can associate with a variety of antigenic peptides non-covalently in vivo and in vitro ${ }^{[14]}$. Recent studies have indicated that gp96 molecules participate in major histocompatibility complex class I-restricted antigen presentation pathway. Immunization of mice with gp96 preparations isolated from cancer cells could elicit a cancer-specific protective $\mathrm{T}$ cell immune response, suggesting the use of gp96 as a therapeutic vaccine against cancers ${ }^{[14]}$. Indeed, HSP-peptide complexes bind to endocytic receptors including CD91 and are taken up by DCs via receptor-mediated endocytosis. Peptides carried on HSP are delivered to the MHC I presentation pathway and are represented on the cell surface in association with MHC class I antigens for recognition by antigen-specific CD8+ $\mathrm{T}$ cells via the TCR and associated molecules. HSP also delivers maturation signals to the DCs and induces the expression of MHC antigens, co-stimulatory molecules (Such as B7, which binds to CD28) and ICAM-1. Simultaneously, the induction of pro-inflammatory cytokines promotes the generation of immune responses to HSP-chaperoned peptides (Figure 1). The induction of cytokines especially IL-12, leads to CTL responses and IFN- $\gamma$ production. Some cytokines including IL- 2 , IFN- $\gamma$ and IFN- $\beta$ stimulate NK-cells to produce IFN- $\gamma$. IFN- $\gamma$ can affect DCs for maturation and more cytokine release such as IL-12 and active CD8+ T cells.

Regarding to previous data, there is no efficient method to obtain high amount of full-length gp96 by in vitro expression. Currently, the yeast Hansenula polymorpha was used as an efficient host for gp96 recombinant protein production. Moreover, the $H$. polymorpha expressed recombinant gp96 could effectively induce HBV-specific CTL response in immunized mice while Escherichia coli-expressed gp96 could not. Thus, these results may provide bases for structure and functional studies of gp96 and thereby potentially accelerate the development of gp96-based vaccines for immunotherapy of cancer or infectious diseases ${ }^{[15]}$.

Active immunotherapy using peptide vaccines is expected to induce specific, cytolytic T cells in the patient, which destroy malignant cells. Although, the identification and low immunogenicity of tumor-specific peptides still remain a serious problem. Gp96-peptide complexes extracted from cancer cells harbor the tumor-specific peptides and are immunogenic, thus offering a tool for active immunization against the tumor. Currently, Vitespen, a commercial HSP-peptide complex vaccine based on tumor-derived gp96, was found to induce an improved survival for subsets of early stage melanoma and kidney cancer patients ${ }^{[16]}$. However, the immunogenicity of gp96 molecules has been attributed to the antigenic peptides associated with them (Figure 1) ${ }^{[14]}$. Previously, a 7-mer HLA-A11-restricted peptide (YVNTNMG) of HBV core Ag (HBcAg 88-94) associated with heat shock protein gp96 was reported in liver tissues of patients with HBV-induced hepatocellular carcinoma (HCC). This peptide was highly homologous to a human HLA-A11-restricted 9-mer peptide 
(YVNVNMGLK) and to a mouse H-2-Kd restricted 9-mer peptide (SYVNTNMGL). To further characterize its immunogenicity, BALB/c mice were vaccinated with the HBV 7-mer peptide. A specific CTL response was induced by the 7-mer peptide ${ }^{[17]}$. To evaluate the adjuvant effect of gp96, mice were co-immunized with gp96 and the 9-mer peptide, and a significant adjuvant effect was observed with gp96. These results clearly demonstrated the potential of gp96 as a possible adjuvant to augment $\mathrm{CTL}$ response against $\mathrm{HBV}$ infection and $\mathrm{HCC}^{[17]}$. In other study, the elevated expression of gastrin releasing peptide (GRP) in various types of cancer suggested that GRP might be a putative target for immunotherapy in neoplastic diseases. Based on six copies of the B cell epitope GRP 18-27 in a linear alignment as an immunogen, several anti-GRP DNA vaccines were designed using different combinations of immune-adjuvants, such as HSP65, tetanus toxoid 830-844 (T), pan HLA-DR-binding epitope (PADRE) (P), and mycobacterial HSP70 407-426 (M), on a backbone of pCR3.1 plasmid vector with eight 50-GACGTT-30 CpG motifs and the VEGF183 signal peptide (VS). Immunization of mice with pCR3.1-VS-HSP65-TP-GRP6-M2 elicited much higher levels of specific anti-GRP antibodies and more effectively inhibited the growth of a GRP-dependent tumor RM-1 in vivo. On the other hand, plasmids encoding for 2HSP70 407-426 showed stronger immune stimulatory potential as well as impressive antitumor activity, suggesting that 2HSP70 407-426 is an efficient molecular adjuvant for developing self-epitope vaccines ${ }^{[18]}$.

Currently, our research group has focused on different vaccine modalities including DNA vaccines, protein vaccines, live vaccines and the combined approaches (e.g., prime-boost vaccines) against HPV infections. Human papillomaviruses (HPV), particularly HPV16, is considered a necessary cause of cervical and oral cancer. Thus, the development of an efficient vaccine against HPV is important for the control of cervical cancer. In our studies, various strategies were applied to enhance DNA vaccine potency including the utilization of adjuvants especially heat shock proteins (e.g., gp96) and/or different delivery systems. In a study, the levels of humoral and cellular immune responses were compared using HPV16 E7 + gp96 co-injection as DNA/DNA and prime-boost (DNA/protein) immunization strategies in C57BL/6 mice model. Assessment of cellular immune responses against E7 antigen indicated that co-delivery of naked DNA E7 + Gp96 plasmid is immunologically more effective than E7 alone and induces Th1 response ${ }^{[1,20]}$.

The purified recombinant NH2-terminal domain of calreticulin (Amino acids 1-180 named vasostatin) inhibited the proliferation of endothelial cells and also angiogenesis in vivo. Compared with other inhibitors of angiogenesis, vasostatin is a small, soluble, and stable molecule that is easy to produce and deliver. As an angiogenesis inhibitor that specifically targets proliferating endothelial cells, vasostatin demonstrated a unique potential for cancer treatment. When vasostatin inoculated into athymic mice, it significantly reduced growth of human Burkitt lymphoma and human colon carcinoma ${ }^{[21]}$. In addition, the researchers have shown that N domain CRT (NCRT) or C-terminal half of HSP70 linked with HPV16 E7 are capable of inducing potent antigen-specific CTL activity in experimental animal models. NCRT and hsp synergistically exhibited significant increases in E7-specific CD8+ T cell responses and notable antitumor effects against E7-expressing tumors. Furthermore, the NCRT/E7/hsp fusion protein generated potent anti-angiogenic effects. These results indicated that NCRT/E7/hsp fusion protein is a promising therapeutic vaccine for treatment of cervical cancer through a combination of antigen-specific immunotherapy and anti-angiogenesis, with possible therapeutic potential in clinical trials ${ }^{[22]}$.

In another study, a fusion protein was designed including heat-shock protein 70 (Hsp70) and the C-terminal 34 amino acids of herpes simplex virus VP22 protein (VP22 $268-301$ ), the former having a peptide binding domain and an ability to be recognized by APCs, and the latter able to achieve cell penetration. Hsp70-VP22 $268-301$ fusion protein was efficiently taken up by mouse dendritic cell (DC) line DC2.4. Electroporation-assisted injection of naked plasmid vector expressing Hsp70-VP22 268-301 into subcutaneous tumors of EG7-OVA, a mouse lymphoma-expressing OVA, significantly increased the survival of mice compared with the same treatment with a plasmid expressing Hsp70. These results indicated a new therapeutic method to induce tumor-specific antitumor immunity without identifying isolating tumor-associated antigens ${ }^{[23]}$. 
Sequential immunization with a recombinant DNA vaccine encoding AFP and heat shock protein70 could generate effective AFP-specific T cell responses and induce definite antitumor effects on AFP-producing tumors, which may be suitable for some clinical testing as a vaccine for HCC ${ }^{[24]}$. In addition, SGN-00101 (HspE7, San Diego, CA) has been known as a novel therapeutic vaccine containing an M. bovis BCG Hsp65 covalently linked to the entire sequence of HPV 16 E7. This trial was designed to evaluate the efficacy and toxicities of HspE7 in women with CIN III. HspE7 demonstrated activity in women with CIN III ${ }^{[25]}$.

Recent studies have shown that another HSP, known as HSP110, exhibits immuno-stimulatory effects when purified from a tumor. HSP110 has been shown to be representative of a recently discovered HSP family, which is expressed in all of the eukaryotic cells. A hallmark of HSP110 is its ability to efficiently bind to and stabilize large protein substrates. As just described, the ability of HSPs to bind short peptides has formed the basis for their use as cancer vaccines. However, HSPs can also bind to and stabilize large proteins. Indeed, the activity of forming natural chaperone complexes with large proteins is an essential component of the HSP function in numerous cellular processes ${ }^{[26]}$. In a study, recombinant HSP110 was non-covalently complexed during heat shock with a recombinant tumor protein antigen in vitro. This natural chaperone complex was then evaluated as an effective vaccine. This vaccine was not be patient specific, as is the case with tumor-derived HSPs/GRPs, but could be applied to any patient with a tumor expressing that protein antigen and did not require a surgical specimen to prepare. In addition, unlike tumor-derived HSPs, where only a small fraction of chaperoned peptide would be expected to be immunogenic, this approach will present to the immune system a highly concentrated tumor protein antigen in a natural chaperone complex with the immunologically active HSP. HER-2/neu was selected as a protein antigen for current study, because it was highly relevant to breast cancer as well as other cancers such as ovarian, prostate, lung, and colon. It was shown that a HSP110-ICD of HER-2/neu chaperon complex, without an adjuvant, was able to elicit both cell-mediated and humoral immune responses against the ICD. Moreover, this natural complex was as efficient as CFA, eliciting an antigen-specific CD8+ T-cell response, both in a CD4+ dependent and in a CD4+ independent manner. Importantly, no anti-HSP110 cell-mediated or humoral immune responses were observed ${ }^{[26]}$.

The other investigation examined the vaccine potential of hsp110 and glucose-regulated protein (grp) 170. At first, it was shown that prior vaccination with hsp110 or grp170 purified from methylcholanthrene- induced fibrosarcoma caused complete regression of the tumor. In a second tumor model, hsp110 or grp170 purified from Colon 26 tumors led to a significant growth inhibition of this tumor. In addition, hsp110 or grp170 immunization significantly extended the life spans of Colon 26 tumor-bearing mice when applied after tumor transplantation. Indeed, a tumor-specific cytotoxic $\mathrm{T}$ lymphocyte response developed in the mice immunized with tumor-derived hsp 110 or grp $170^{[27]}$. Furthermore, treatments of the mice with bone marrow-derived dendritic cells pulsed with these two proteins from tumor elicited a strong anti-tumor response. Finally, the experiments showed that mild, fever-like hyperthermic conditions enhance the vaccine efficiency of hsp110, but not grp170. These studies indicated that hsp110 and grp170 can be used in cancer immunotherapy and fever-level hyperthermia can significantly enhance the vaccine efficiency of some hsps ${ }^{[27]}$.

HSP 110 has been also used as a chaperone immuno-adjuvant to enhance the immune response to HPV16 E7-derived CTL epitope E7 (49-57) in a mouse model. Inoculation of the mHSP110-E7 (49-57) complex was capable of priming strong epitope-specific immune response and IFN $\gamma$ production. More importantly, immunization of this complex also significantly inhibited the growth of established tumors and prolonged the survival time of the tumor-bearing animals. Results also showed that immunization with this complex completely protected mice against subsequent challenge with tumor cells ${ }^{[28]}$.

As known, the heat shock protein (HSP)/peptides (HSP/Ps) complexes can induce both adaptive and innate immune responses (Figure 1). Treatment with HSP/Ps in tumor-bearing mice and cancer patients has revealed anti-tumor immune activity. The authors have indicated that immunotherapy strategies by vaccination with a mixture of HSP/Ps (mHSP/Ps, HSP60, HSP70, Gp96 and HSP110) enhance with cyclophosphamide (CY) and IL-12. In mice vaccinated with this enhanced vaccine (mHSP/Ps and CY plus IL-12), 80\% showed tumor regression and long-term survival, and tumor 
growth inhibition rate was $82.3 \%$ (30 days); whereas, all controls died within 40 days. After vaccination, lymphocytes and polymorphonuclear leukocytes infiltrated into the tumors of treated animals, but no leukocytes infiltrated into the tumors of control mice. All ratios of natural killer cells, CD8+, and IFN- $\gamma$-secreting cells were increased in the test group, and also tumor-specific cytotoxic $\mathrm{T}$ lymphocyte activity was increased. The regimen may be a promising therapeutic agent against tumors ${ }^{[29]}$.

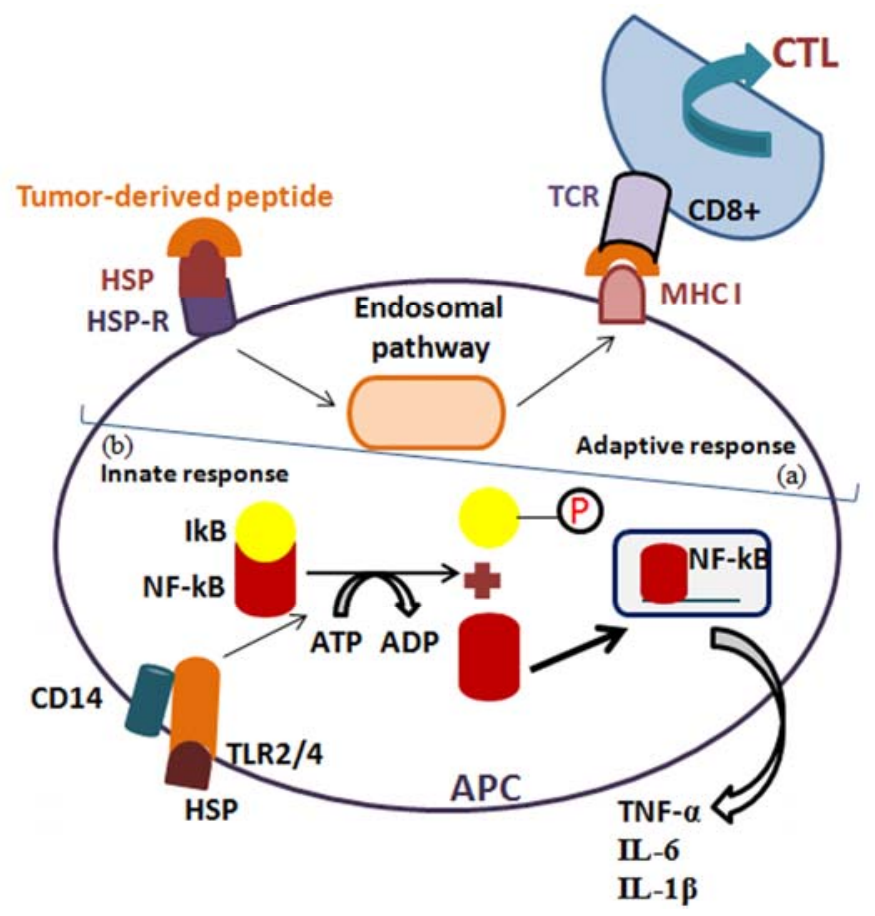

Figure 1. Immunological properties of HSPs: (a) Peptide-carrier function of HSPs: HSP-chaperoned antigenic peptides derived from tumor cells are internalized by endocytosis via HSP receptors (HSP-R). After uptake and processing, the peptides are represented on MHC class I molecules of the APC and thus induce a CD8+ T cell response against cancer; (b) Cytokine-inducing effects of HSP70: In the absence of antigenic, tumor-derived peptides, HSPs engage signaling receptors (e.g., TLR2/4 plus CD14) triggering NF-KB activation. The APCs release pro-inflammatory cytokines (e.g., IL-1 $\beta$, IL-6, TNF- $\alpha$ ) leading to a non-specific stimulation of the innate immune system.

Finally, it is worthwhile to know HSPs structure and function due to usage as a novel vaccine adjuvant. For instance, Mycobacterium tuberculosis HSP70 (mHSP70) consists of three functionally distinct domains: An N-terminal $44 \mathrm{kDa}$ ATPase portion (Amino acids 1-358), followed by an $18 \mathrm{kDa}$ peptide-binding domain (Amino acids 359-494) and a 10 $\mathrm{kDa}$ fragment (Amino acids 495-610). The C-terminal portion (Amino acids 359-610) was proven to stimulate the production of CC chemokines, IL-12, TNF- $\alpha$, NO and the maturation of dendritic cells (DCs). In addition, a cytotoxic cell-inducing function was demonstrated in the ATPase portion (Amino acids 161-370) of mHSP70 ${ }^{[30]}$.

\section{Conclusion}

One major challenge in developing effective vaccines is to design a vaccine that can induce effective immune responses to the desired antigen with no or very limited side effects. The immunogenicity can be improved by using appropriate carriers and adjuvant molecules. Heat shock proteins (HSPs) are some of the most conserved proteins present in all prokaryotes and eukaryotes which posses highly immunogenic effect and function as adjuvants that may play a crucial role in integrating innate and adaptive immunity. They are effective biomarkers for carcinogenesis in some tissues and show the degree of differentiation of certain cancers. In addition, the levels of HSP and anti-HSP antibodies in the serum of cancer 
patients are useful in tumor diagnosis. HSP may also predict the response to some anticancer treatments. Implication of HSP in tumor progression and response to therapy has led to its successful targeting in therapy by two major strategies, such as: a) Pharmacological modification of HSP expression or molecular chaperone activity and b) Use of HSPs as adjuvants to present tumor antigens to the immune system. Study of HSP in cancer is still in its early stages, and needs to obtain more information at the cell and molecular levels involved with tumor growth, invasiveness and metastasis. Such studies will be necessary in targeting HSPs in cancer therapy and knowing their crucial immunological functions.

\section{Conflict of interest}

The authors declare that there is no conflict of interest statement.

\section{References}

[1] Gong J, Zhang Y, Durfee J et al. A heat shock protein 70-based vaccine with enhanced immunogenicity for clinical use. The Journal of Immunology 2010;184(1):488-96. PMid:19949080 http://dx.doi.org/10.4049/jimmunol.0902255

[2] Pathan MM, Latif A, Das H, Siddiquee GM, Khan MJZ. Heat Shock Proteins and their clinical Implications. Veterinary World 2010;3(12):558-60.

[3] Barnes JA, Dix DJ, Collins BW, Luft C, Allen JW. Expression of inducible Hsp70 enhances the proliferation of MCF-7 breast cancer cells and protects against the cytotoxic effects of hyperthermia. Cell Stress Chaperones 2001;6(4):316-25. http://dx.doi.org/10.1379/1466-1268(2001)006<0316:EOIHET>2.0.CO;2

[4] Kim LS, Kim JH. Heat shock protein as molecular targets for breast cancer therapeutics. J Breast Cancer 2011;14(3):167-174. PMid:22031796 http://dx.doi.org/10.4048/jbc.2011.14.3.167

[5] Urushibara M, Kageyama Y, Akashi T et al. HSP60 may predict good pathological response to neo-adjuvant chemo-radiotherapy in bladder cancer. Jpn J Clin Oncol. 2007;37(1):56-61. PMid:17095522 http://dx.doi.org/10.1093/jjco/hyl121

[6] Laudanski K, Wyczechowska D. The distinctive role of small heat shock proteins in oncogenesis. Archivum Immunologiae et Therapiae Experimentalis 2006;54(2):103-11. PMid:16648970 http://dx.doi.org/10.1007/s00005-006-0013-3

[7] Peng RQ, Chen YB, Ding Y et al. Expression of calreticulin is associated with infiltration of T-cells in stage IIIB colon cancer. World J Gastroenterol. 2010;16(19):2428-34. PMid:20480531 http://dx.doi.org/10.3748/wjg.v16.i19.2428

[8] Lwin ZM, Guo C, Salim A et al. Clinicopathological significance of calreticulin in breast invasive ductal carcinoma. Mod Pathol. 2010;23(12):1559-66. PMid:20834237 http://dx.doi.org/10.1038/modpathol.2010.173

[9] Toquet C, Jarry A, Bou-Hanna C et al. Altered Calreticulin expression in human colon cancer: Maintenance of Calreticulin expression is associated with mucinous differentiation. Oncol Rep. 2007;17(5):1101-07. PMid:17390051

[10] Tosato G, Yao L, Pike SE. Calreticulin and tumor suppression. Madame Curie Bioscience Database, Landes Bioscience 2000; Bookshelf ID: NBK6190.

[11] Liu R, Gong J, Chen J et al. Calreticulin as a potential diagnostic biomarker for lung cancer; Cancer Immunology Immunotherapy 2011. PMCid:3080434 http://dx.doi.org/10.1007/s00262-011-1146-8

[12] Dudeja V, Vickers SM, Saluja AK. The role of heat shock proteins in gastrointestinal diseases. Gut 2009;58:1000-09. PMid:19520890 http://dx.doi.org/10.1136/gut.2007.140194

[13] Udono H, Srivastava PK. Comparison of tumor-specific immunogenicities of stress-induced proteins gp96, hsp90 and hsp70. J Immunol. 1994;152(11):5398-403. PMid:8189059

[14] Peibin Y, Shude Y, Changzhi H. Heat shock protein gp96 and cancer immunotherapy. Chin Med Sci J. 2002;17(4):251-6. PMid:12901515

[15] Li Y, Song H, Li J et al. Hansenula polymorpha expressed heat shock protein gp96 exerts potent T cell activation activity as an adjuvant. J Biotechnol. 2011;151(4):343-49. PMid:21167226 http://dx.doi.org/10.1016/j.jbiotec.2010.12.006

[16] Randazzo M, Terness P, Opelz G, Kleist C. Active-specific immunotherapy of human cancers with the heat shock protein Gp96-revisited. International Journal of Cancer 2012;130(10):2219-31. PMid:22052568 http://dx.doi.org/10.1002/ijc.27332

[17] Li H, Zhou M, Han J et al. Generation of murine CTL by a Hepatitis B Virus-specific peptide and evaluation of the adjuvant effect of heat shock protein glycoprotein 96 and its terminal fragments. The Journal of Immunology 2005;174:195-204. PMid:15611241

[18] Lu Y, Mekoo DJL, Ouyang K et al. Strong humoral response elicited by a DNA vaccine targeting gastrin-releasing peptide with optimized adjuvants inhibits murine prostate carcinoma growth in vivo. Endocr Relat Cancer 2009;16:1171-84. PMid:19648182 http://dx.doi.org/10.1677/ERC-09-0058 
[19] Bolhassani A, Zahedifard F, Taghikhani M, Rafati S. Enhanced immunogenicity of HPV16E7 accompanied by Gp96 as an adjuvant in two vaccination strategies. Vaccine 2008;26:3362-70. PMid:18471945 http://dx.doi.org/10.1016/j.vaccine.2008.03.082

[20] Bolhassani A, Zahedifard F, Taslimi Y, Taghikhani M, Nahavandian B, Rafati S. Antibody detection against HPV16 E7 \& GP96 fragments as biomarkers in cervical cancer patients. Indian J Med Res. 2009;130(5):533-41. PMid:20090101

[21] Pike SE, Yao L, Jones KD et al. Vasostatin, a Calreticulin fragment, inhibits angiogenesis and suppresses tumor growth. J Exp Med. 1998;188(12):2349-56. PMid:9858521 http://dx.doi.org/10.1084/jem.188.12.2349

[22] Liu B, Ye D, Song X et al. A novel therapeutic fusion protein vaccine by two different families of heat shock proteins linked with HPV16 E7 generates potent antitumor immunity and anti-angiogenesis. Vaccine 2008;26(10):1387-96. PMid:18272260 http://dx.doi.org/10.1016/j.vaccine.2007.12.034

[23] Nishikawa M, Otsuki T, Ota A et al. Induction of tumor-specific immune response by gene transfer of Hsp70-cell-penetrating peptide fusion protein to tumors in mice. Mol Ther 2010;18(2):421-28. PMid:19724264 http://dx.doi.org/10.1038/mt.2009.203

[24] Wang XP, Liu GZ, Song AL, Li HY, Liu Y. Antitumor immunity induced by DNA vaccine encoding alpha-fetoprotein/heat shock protein 70. World J Gastroenterol. 2004;10(21):3197-200. PMid:15457574

[25] Einstein MH, Kadish AS, Burk RD et al. Heat shock fusion protein-based immunotherapy for treatment of cervical intraepithelial neoplasia III. Gynecol Oncol. 2007;106(3):453-60 PMid:17586030 http://dx.doi.org/10.1016/j.ygyno.2007.04.038

[26] Manjili MH, Henderson R, Wang XY et al. Development of a recombinant HSP110-HER-2/neu vaccine using the chaperoning properties of HSP110. Cancer Res. 2002;62(6):1737-42. PMid:11912148

[27] Wang XY, Kazim L, Repasky EA, Subjeck JR. Characterization of heat shock protein 110 and glucose-regulated protein 170 as cancer vaccines and the effect of fever-range hyperthermia on vaccine activity. J Immunol. 2001;166(1):490-97. PMid:11123328

[28] Ren F, Xu Y, Mao L et al. Heat shock protein 110 improves the antitumor effects of the cytotoxic T lymphocyte epitope E749-57 in mice. Cancer Biol Ther. 2010;9(2):134-41. http://dx.doi.org/10.4161/cbt.9.2.10391

[29] Guo QY, Yuan M, Peng J et al. Antitumor activity of mixed heat shock protein/ peptide vaccine and cyclophosphamide plus interleukin-12 in mice sarcoma. J Exp Clin Cancer Res. 2011;30:24. http://dx.doi.org/10.1186/1756-9966-30-24

[30] Ebrahimi SM, Tebianian M. Role of mycobacterial heat shock protein 70 (mHSP70) as genetic vaccine adjuvants. World Applied Sciences Journal 2011;14(10):1569-75. 